\title{
PERFORMANCE DE COAGULANTES ORGÂNICOS E INORGÂNICOS POR MEIO DE DIAGRAMA DE COAGULAÇÃO EM ÁGUAS NATURAIS
}

\section{PERFORMANCE OF ORGANIC AND INORGANIC COAGULANTS BY COAGULATION DIAGRAM IN NATURAL WATER}

Thyago Nóbrega Silveira.

Engenheiro Sanitarista e Ambiental, Universidade Estadual da Paraíba. (thyagonobrega1996@gmail.com)

Maria Gabriella Negromonte Barbosa.

Graduanda em Engenharia Sanitária em Ambiental Universidade Estadual da Paraíba.

(mariagabriellanegromonte@yahoo.com.br)

Lucas Alves Batista Pequeno.

Graduando em Engenharia Sanitária e Ambiental, Universidade Estadual da Paraíba.

(lucaspequeno alves@hotmail.com)

\section{Whelton Brito Dos Santos.}

Mestre em Engenharia Civil e Ambiental, Universidade Federal de Campina Grande. Doutorando em Recursos Naturais /UFCG. (wheltonbrt@gmail.com)

Weruska Brasileiro Ferreira.

Doutora em Engenharia Química, Universidade Federal de Campina Grande. (weruskabrasileiro2016@gmail.com)

\section{Resumo}

Este estudo teve como objetivo avaliar a performance dos coagulantes orgânicos e inorgânicos por meio de diagramas de coagulação, verificando as melhores condições de $\mathrm{pH}$ e dosagem para remoção de cor e turbidez, utilizando a água bruta do reservatório Epitácio Pessoa (Boqueirão, Paraíba). Foram realizados ensaios de coagulação/floculação/sedimentação em aparelho jar test, com o emprego de coagulantes orgânicos (Tanfloc SG e Tanfloc SL) e inorgânicos (sulfato de alumínio e cloreto férrico). A faixa de pH utilizada para a construção dos diagramas de coagulação para os coagulantes inorgânicos e orgânicos foram de 4,5 a 9,5 e 5,5 a 9,0, respectivamente, variando as dosagens dos coagulantes aplicadas na água. Com base nos diagramas de coagulação, observou-se que os flocos obtidos com os coagulantes orgânicos apresentaram velocidades de sedimentação maiores em comparação aos coagulantes inorgânicos, proporcionando melhor remoção dos sólidos suspensos, obtendo-se na etapa da decantação valores de turbidez inferiores a 0,5 uT em amplas faixas de $\mathrm{pH}$.

Palavras-chave: Diagramas de Coagulação. Coagulantes orgânicos. Coagulantes Inorgânicos.

\section{Abstract}

This study had as objective, to evaluate the performance of organic and inorganic coagulants, through the coagulation diagrams, verifying the best $\mathrm{pH}$ and dosage conditions for the removal of color and turbidity of the raw water from the Epitácio Pessoa reservoir (Boqueirão, Paraíba). Coagulation / flocculation / sedimentation assays were carried out in the flocculation jar tester equipment, using organic coagulating agents (Tanfloc SG and Tanfloc SL) and inorganic (aluminum sulphate and ferric chloride). The pH range used for the construction of the coagulation diagrams for the organic and inorganic coagulant systems, was from 4.5 to 9.5 and from 5.5 to 9.0 respectively, varying the dosages of the coagulants applied in the water. Based on the results of the coagulation, it was observed that the obtained flocs with the organic agents, presented higher sedimentation rate in comparison to the inorganic coagulants, providing better removal of the suspended solids, obtaining in the decantation stage, turbidity values lower than $0,5 \mathrm{uT}$ in wide ranges of $\mathrm{pH}$.

Key-words: Coagulation diagrams. Organic coagulants. Inorganic coagulants. 


\section{INTRODUÇÃO}

A água bruta pode ser definida como aquela sem tratamento e imprópria ao consumo humano. As impurezas presentes na água bruta podem ser classificadas por suas características químicas, como $\mathrm{pH}$, alcalinidade,

acidez, dureza, cloretos, ferro, entre outras, e por suas características físicas, em forma de dispersão coloidal (SANTOS, 2014). Como principais parâmetros de caracterização física das águas, destacam-se a turbidez e a cor, que são os mais utilizados no monitoramento da água produzida nas estações de tratamento.

Para o tratamento da água, os sais de alumínio e de ferro são muito utilizados como agentes coagulantes no processo (FERRARI et al., 2012; PAVANELLI, 2001). Estes coagulantes inorgânicos a base de sais metálicos, quando em contato com água, formam reações de hidrólise, produzindo espécies de cargas positivas com capacidade de reduzir significativamente 0 potencial zeta do meio aquoso (FARIAS; FERREIRA; FERRO, 2014).

Apesar do baixo custo comercial e eficiência, estes coagulantes inorgânicos apresentam como principal dificuldade do processo a geração de lodo inorgânico, de difícil manuseio por parte das empresas em função de seu volume e do elevado teor de umidade, além de deixar traços químicos na água após o tratamento, que podem gerar malefícios à saúde (CRUZ et al., 2005; DI BERNARDO; SABOGAL PAZ, 2008; MCLACHLAN, 1995).

Atualmente, devido à degradação da qualidade da água dos reservatórios superficiais e ao aumento da rigorosidade da legislação, tem sido necessária a utilização de outros produtos e tecnologias para auxiliar no processo de coagulação, aplicando em conjunto com os coagulantes inorgânicos, tais como pré-oxidantes, alcalinizantes, polímeros inorgânicos e orgânicos, uso de membranas filtrantes, adsorventes, entre outros, a fim de alcançar o padrão de potabilidade (BATAGHIN; COSTA; TAKAGAKI, 2017; OLIVEIRA; MIERZWA, 2011; WESTPHALEN; CORÇÃO; BENETTI, 2016).

De acordo com a TANAC, empresa produtora de coagulantes orgânicos, o Tanfloc $\mathrm{SG} \circledast$ e o Tanfloc SL® (utilizados neste estudo) são polímeros orgânicos catiônicos de baixo peso molecular de origem vegetal, utilizados como coagulantes/floculantes, auxiliares de coagulação no tratamento de águas em geral. Atuam em partículas coloidais, neutralizando cargas e formando pontes entre essas partículas, sendo este processo responsável pela formação de flocos. Os coagulantes naturais apresentam algumas vantagens em relação aos inorgânicos, sendo biodegradáveis e não tóxicos, e produzem lodo em menor quantidade e com menor teor de metais.

Diversas estações de tratamento de água no Brasil e no mundo já utilizam os coagulantes orgânicos como alternativa para o tratamento de água e efluentes, atingindo os padrões de potabilidade. Além disso, o lodo gerado em decorrência do tratamento apresenta-se dentro das faixas normais de especificação, apontando ausência de sais de alumínio e ferro em sua composição e facilitando o manejo e tratamento (MANGRICH et al., 2014).

Segundo Piantá (2008), o uso alternativo de coagulantes orgânicos em detrimento dos inorgânicos pode gerar um aumento dos custos no tratamento de água, uma vez que, comercialmente, o produto é mais caro e seriam necessárias alterações estruturais na estação para aplicação do coagulante. Porém, não foi levada em consideração para essa afirmação a redução dos custos com a não aplicação de alcalinizantes na pré-coagulação, além de simplificar o processo de tratamento, sendo necessário menor consumo de energia.

No processo de tratamento da água, a coagulação química é uma das primeiras etapas pelas quais a água é submetida. Assim, o bom funcionamento desta etapa está diretamente relacionado ao sucesso das etapas posteriores, sendo de extrema importância para o tratamento da água (EDZWALD, 2011). Para que se atinja o desempenho esperado na etapa de coagulação, é necessária a correta seleção do coagulante e sua dosagem, bem como do $\mathrm{pH}$ apropriado para sua utilização. Para isso, são realizados estudos de tratabilidade em laboratório (DI BERNARDO; DANTAS; VOLTAN, 2011).

Para a avaliação da eficiência do tratamento, muitas vezes utilizam-se diagramas de coagulação, a fim de perceber as melhores faixas de $\mathrm{pH}$ e dosagem dos coagulantes (AMIRTHARAJAH; MILLS, 1982; BARTIKO; JULIO, 2015; BOMFIM, 2015; PAVANELLI, 2001; SILVEIRA, 2018). Estes exibem de forma clara e autoexplicativa a eficiência dos coagulantes, além de servirem de modelo para tratamento de água que apresentem características semelhantes à água estudada. 
Portanto, o objetivo deste trabalho foi utilizar como ferramenta avaliativa, para ensaios de tratabilidade em escala laboratorial por meio de jar test, os diagramas de coagulação, analisando a remoção dos parâmetros cor e turbidez, verificando 0 desempenho de coagulantes comerciais orgânicos e inorgânicos no tratamento de águas naturais.

\section{METODOLOGIA}

\subsection{Caracterização da área de estudo}

Os ensaios de coagulação/lloculação/decantação foram realizados em aparelho jar test com água bruta do reservatório superficial Epitácio Pessoa, popularmente conhecido como Boqueirão. O reservatório integra o sistema de abastecimento de água de Campina Grande (SAACG) e de mais 18 municípios, abastecendo, aproximadamente 1,2 milhão de pessoas, sendo este gerido pelo Departamento Nacional de Obras Contra a Seca (DNOCS) (MENESES, 2011).

O reservatório está localizado na Bacia do Rio Paraíba (Figura 1), na porção do alto curso desta bacia, na região do semiárido brasileiro, que possui um embasamento cristalino, gerando dureza e alcalinidade elevadas na água, o que limita o seu uso na indústria e na irrigação (PARAÍBA, 2006). A bacia apresenta média pluviométrica de $600 \mathrm{~mm} / \mathrm{ano}$, o que representa uma região com grande severidade para abastecimento de água (SANTOS, 2014).

As grandes derivações de água para fins de abastecimento, agricultura e industrial, e um longo período de estiagem, resultaram em drástica redução do volume do reservatório, atingindo o volume crítico de $2,9 \%$, em abril de 2017. Frente a essa situação, foi necessária a adoção de medidas emergenciais, a fim de suprir a demanda de água e controlar um possível desabastecimento do sistema Boqueirão (grande sistema de abastecimento que o reservatório faz parte), sendo, então, agilizadas as obras do eixo leste da transposição do Rio São Francisco, para levar as águas do rio ao reservatório Epitácio Pessoa. No período de execução desta pesquisa, o reservatório ainda estava sendo recarregado exclusivamente pelas as águas do Rio São Francisco.

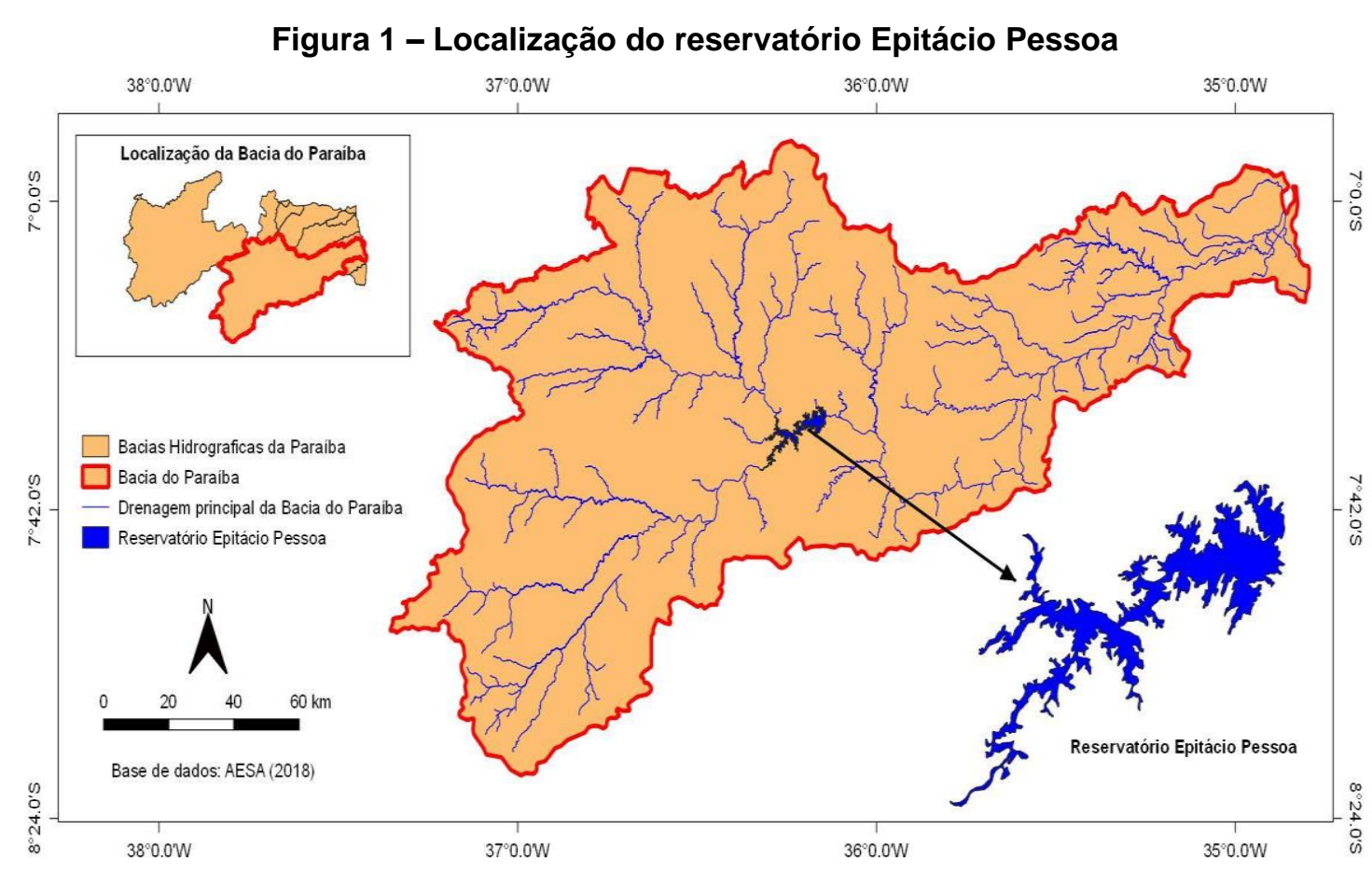

Fonte: Adaptada de GEOPORTAL AESA, 2018.

\subsection{Coleta das amostras}

As amostras de água bruta foram coletadas na chegada da Estação de Tratamento de água de Gravatá (ETA-Gravatá), que opera em ciclo completo, utilizando o sulfato de alumínio na etapa da coagulação. As coletas foram realizadas com uma frequência semanal no período de 23 de janeiro a 16 de fevereiro de 2018. Cada coleta correspondeu a um ensaio, e para cada ensaio 
era aplicado um agente coagulante diferente com variações de $\mathrm{pH}$, sendo utilizados, para este fim, dez recipientes de vinte litros, totalizando duzentos litros de água.

No período de coleta, o reservatório encontrava-se em situação crítica, abaixo do nível mínimo de operação, $11 \%$ do seu volume, quando estava recebendo águas do eixo leste da transposição do Rio São Francisco.

\subsection{Ensaios de laboratório}

Os ensaios foram realizados em um jar test Poli Control $\AA$, modelo Floc Control III, com a aplicação de quatro coagulantes: dois inorgânicos - Sulfato de Alumínio e o Cloreto Férrico, produtos comerciais fornecidos por empresa de saneamento, hidratados; e dois orgânicos à base de Tanino - Tanfloc SL ${ }^{\circledR}$ e o Tanfloc SG ${ }^{\circledR}$.

Foram realizados quatro ensaios distintos, sendo um ensaio para cada coagulante, e cada ensaio gerando dois diagramas de coagulação: um para a turbidez e outro para a cor aparente. Para cada ensaio, efetuava-se cerca de 80 testes de tratabilidade, com variação do $\mathrm{pH}$ da água bruta e da dosagem de coagulante. Os Ensaios 1, 2, 3 e 4 correspondem aos ensaios que utilizaram como agente coagulante o sulfato de alumínio, cloreto férrico, Tanfloc SG $®$ e Tanfloc $\mathrm{SL} \circledast$, respectivamente.

Anteriormente a cada ensaio, a turbidez e a cor aparente das amostras de água bruta em estudo foram determinadas, utilizando medidores nefelométricos da Poli Control $®$ (Aquacolor Cor e Turbidímetro AP2000), a fim de comparação dos resultados pós-ensaio, sendo este valor também utilizado para delinear um contorno dos diagramas de coagulação formados. Para $0 \mathrm{pH}$, foram utilizados medidores portáteis da KASVI ${ }^{\circledR}$, modelo K39-0014PA.

A faixa de $\mathrm{pH}$ utilizada para a execução dos ensaios para os coagulantes inorgânicos e orgânicos foram de 4,5 a 9,5 e 5,5 a 9,0, respectivamente, variando em intervalos de 0,5 . Estas faixas foram definidas de acordo com a Portaria de consolidação do Ministério da Saúde (MS) n 5/2017, Anexo XX, a qual recomenda que a água para abastecimento humano esteja com pH compreendido entre 6,0 e 9,5 (BRASIL, 2017). A resolução 357/2005 do Conselho Nacional do Meio Ambiente (CONAMA) também estabelece que $\mathrm{o} \mathrm{pH}$ de um manancial apto para 0 abastecimento humano precisa estar compreendido entre 6,0 e 9,0 (BRASIL, 2005).
Para a alteração do $\mathrm{pH}$, foram utilizadas soluções de ácido clorídrico $(0,5 \%)$ e hidróxido de sódio $(0,5 \%)$, sendo toleradas variações de $\pm 0,1 \mathrm{no} \mathrm{pH}$ da água.

As dosagens de coagulante inorgânico aplicadas na água bruta variaram a cada ensaio de 10 a $80 \mathrm{mg} \mathrm{L}^{-1}$, com escalonamento de $10 \mathrm{mg}$ $\mathrm{L}^{-1}$. Já os orgânicos variaram de 5 a $50 \mathrm{mg} \mathrm{L}^{-1}$, com escalonamento de $5 \mathrm{mg} \mathrm{L}^{-1}$. Estes valores foram definidos a partir de trabalhos anteriormente efetuados com os coagulantes estudados (BARTIKO; JULIO, 2015; BOMFIM, 2015; FERRARI et al., 2012; MORAES et al., 2007; NEPOMUCENO, 2016).

As soluções dos agentes coagulantes foram preparadas a concentração de $1 \%\left(10 \mathrm{mg} \mathrm{mL}^{-1}\right)$ a partir do produto comercial concedido pelas empresas fabricantes. A partir disso, utilizando a Equação 1, definiram-se os volumes da solução a ser adicionada nos jarros.

$$
V=\frac{D \times V_{\text {Jar }}}{c_{\text {SnI }}}
$$

onde: V: Volume da solução do produto químico adicionado $(\mathrm{mL})$; D: Dosagem do produto químico resultante no jarro ( $\left.\mathrm{mg} \mathrm{L}^{-1}\right)$; $\mathrm{V}_{\text {jar: }}$ Volume do jarro do equipamento jar test (L), sendo o volume do jarro utilizado de 2L; $\mathrm{C}_{\text {sol }}$ : Concentração da solução preparada do produto químico (mg mL-1).

A velocidade angular adotada para simular a unidade de mistura rápida foi de 600 RPM (1400 $\left.\mathrm{s}^{-1}\right)$, com tempo de detenção hidráulica de 10 segundos. As rotações de mistura lenta, por sua vez, foram de 70, 40 e $20 \operatorname{RPM}\left(70,30\right.$ e $\left.10 \mathrm{~s}^{-1}\right)$, com tempo de floculação de 7, 7, 5 minutos, respectivamente. $O$ tempo de decantação foi de 2 minutos e 12 segundos, o que caracteriza uma velocidade de sedimentação adotada de $3 \mathrm{~cm}$ $\min ^{-1}$, já que o ponto de coleta era a $7 \mathrm{~cm}$ do nível máximo de água no jarro. Os valores foram definidos após diversos estudos de tratabilidade realizados pela equipe de laboratório, adaptados da metodologia utilizada por Di Bernardo, Dantas e Voltan (2011).

Após o término das etapas de coagulação/floculação/decantação, para os coagulantes estudados, foi coletada uma amostra de aproximadamente $100 \mathrm{~mL}$ de água decantada de cada jarro do jar test, que, em seguida, foram analisadas em função da remoção de cor aparente e turbidez, utilizando os equipamentos descritos anteriormente, a fim de verificar a eficiência do procedimento. As medições dos 
parâmetros foram feitas em triplicatas, sendo o valor médio empregado na construção dos diagramas.

\subsection{Diagramas de coagulação}

Para a construção dos diagramas de coagulação, utilizou-se o Software MiniTab 17, elaborando gráficos de contorno, com isocurvas de cor e turbidez para cada coagulante utilizado. Os intervalos empregados para determinação das isolinhas levaram em consideração os valores de cor aparente e turbidez da água bruta, valores de turbidez recomendados por Ferrari et al. (2012) antes do processo de filtração (turbidez $<3$ UNT, visando um maior tempo de carreira dos filtros), além do valor máximo de cor permitida pela legislação brasileira para distribuição de água, segundo a Portaria de Consolidação n 5/2017 do Ministério da Saúde, Anexo XX (15 uH) (BRASIL,
2017).

\section{RESULTADOS E DISCUSSÃO}

Os parâmetros cor aparente e turbidez, das águas utilizadas nos ensaios para a construção dos diagramas de coagulação, estão apresentados na Tabela 1. Estes valores obtidos foram também utilizados na construção dos diagramas de coagulação, para se verificar se houve ou não remoção dos sólidos, atestando, assim, a eficiência de atuação dos coagulantes e velocidade de sedimentação dos flocos. Para obtenção dos valores de cor aparente e turbidez da água bruta, foram feitas diversas leituras dos recipientes de coleta, quando foi tomado um valor médio das amostras lidas para ser utilizado como referência comparativa e demonstrativa na construção do diagrama.

Tabela 1 - Caracterização da água bruta utilizada nos ensaios

\begin{tabular}{ccccccccc}
\hline & \multicolumn{2}{c}{ Ensaio 1 } & \multicolumn{3}{c}{ Ensaio 2 } & \multicolumn{2}{c}{ Ensaio 3 } & \multicolumn{2}{c}{ Ensaio 4 } \\
\hline & Média & $\sigma_{\mathrm{n}-1}$ & Média & $\sigma_{\mathrm{n}-1}$ & Média & $\sigma_{\mathrm{n}-1}$ & Média & $\sigma_{\mathrm{n}-1}$ \\
\hline Cor Aparente (uC) & 18,8 & 0,5556 & 23,5 & 1,3111 & 32,5 & 1,1111 & 24,0 & 1,6444 \\
Turbidez (UNT) & 0,64 & 0,0444 & 0,82 & 0,0911 & 0,84 & 0,0444 & 0,92 & 0,0444 \\
\hline
\end{tabular}

Fonte: Dados da pesquisa.

Como podem ser observados, no decorrer dos quatro ensaios, os valores de cor aparente e turbidez aumentaram de forma discreta. Esse aumento discreto pode ser explicado pela alteração do volume da água, devido ao aporte de forma gradual e contínua $\left(3,51 \mathrm{~m}^{3} \mathrm{~s}^{-1}\right)$ de água ao reservatório pela transposição do Rio São Francisco, onde não há carreamento de muitos sólidos em suspensão para o interior do manancial, justificando as discretas mudanças nos valores encontrados, uma vez que a captação da água para a estação de tratamento é distante do ponto de recarga do reservatório. Quando a recarga do manancial é feita por água da chuva, além de possuir uma maior vazão, muitos sedimentos são carreados devido ao grande volume de água, por conta da estrutura do solo da bacia, elevando bruscamente os valores de cor aparente e turbidez.

A Figura 2 apresenta os diagramas de coagulação, para os parâmetros de cor aparente e turbidez, para o agente coagulante Sulfato de Alumínio.

Com a aplicação do sulfato de alumínio, a turbidez foi superior à da água bruta (Figura 2.B), havendo uma redução da cor em faixa de $\mathrm{pH}$ em torno de 9,5 e uma dosagem de $30 \mathrm{mg} \mathrm{L}^{-1}$ (Figura 2.A). Levando em consideração apenas a turbidez, verificou-se que os melhores resultados foram apresentados com baixas concentrações de coagulante, com pH acima de aproximadamente 8,0 , indicando uma prevalência do mecanismo de adsorção-desestabilização na coagulação, uma vez que a faixa de pH ideal para o uso de sulfato de alumínio é restrita, aproximando-se do pH neutro (BARTIKO; JULIO, 2015; BOMFIM, 2015; PAVANELLI, 2001).

Shen (2005) afirma que quanto menor a turbidez natural da água, mais difícil se torna a remoção dela através do sulfato de alumínio, sendo necessárias altas concentrações do coagulante, utilizando o mecanismo de coagulação por varredura; mecanismo este que não foi possível alcançar com as dosagens estudadas.

Os diagramas de coagulação para o Ensaio 2, com cloreto férrico, podem ser observados na Figura 3.

No uso do cloreto férrico, é possível observar que, semelhante ao sulfato de alumínio, os valores dos parâmetros foram superiores aos da água bruta, com destaque à cor (Figura 3.A), que 
teve um grande aumento devido à coloração alta do próprio cloreto férrico, sendo evidente, principalmente, em altas concentrações deste, obtendo cor aparente acima de $400 \mathrm{uC}$. Os melhores resultados para turbidez (Figura 3.B) foram obtidos em uma ampla faixa de $\mathrm{pH}, 6,0$ a 9,5 , com concentrações entre 10 e $70 \mathrm{mg} \cdot \mathrm{L}^{-1}$, obtendo-se valores entre 0,82 e 3,0 UNT, sendo preferível a utilização de baixas concentrações devido à cor associada a altas concentrações. Isto é compatível com a referência bibliografia que afirma que este coagulante trabalha em largas faixas de pH (SILVA et al., 2016). Para esta faixa, prevalece o mecanismo de adsorçãodesestabilização, pois na observância dos dados absolutos, percebe-se que há menor turbidez em concentrações menores.

\section{Figura 2 - Diagramas de coagulação para o Ensaio 1 (Sulfato de Alumínio), contendo isocurvas de cor aparente $(A)$ e turbidez (B)}
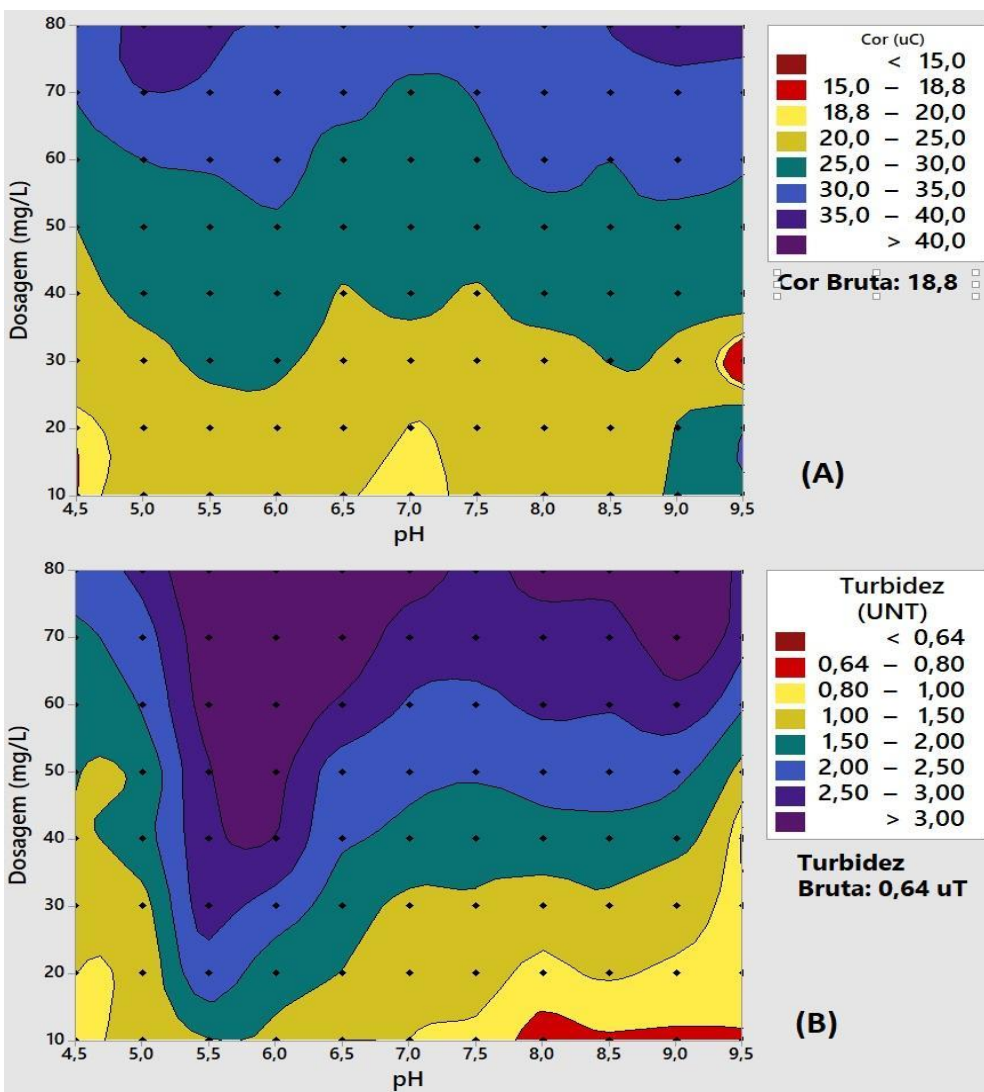

Fonte: Elaborada pelos autores.

Nepomuceno (2016) alcançou valores de remoção de turbidez de até $92 \%$ com o cloreto férrico em águas de elevada turbidez com dosagem de $10 \mathrm{mg} \mathrm{L}^{-1}$ de coagulante, utilizando um maior tempo de floculação e o dobro da velocidade de sedimentação apresentada neste estudo. Como é possível perceber nos diagramas, nenhum ponto apresentou redução de turbidez ou cor aparente após o ensaio. Segundo Shen (2005), os sais de alumínio e ferro possuem dificuldade de atuar em águas de baixa turbidez, sendo necessária a utilização de outros produtos no tratamento, tais como oxidantes e polímeros.

A Figura 4 apresenta os diagramas de coagulação resultantes do Ensaio 3 , com a aplicação do Tanfloc SG.
Os diagramas dos ensaios com Tanfloc SG apresentaram redução nos valores de cor e turbidez. Conforme pode ser observado na Figura 4.A, a cor aparente teve uma redução em $\mathrm{pH}$ entre 5,5 e 6,0 em baixa concentração do coagulante $\left(5\right.$ e $\left.10 \mathrm{mg} \mathrm{L}^{-1}\right)$. Em relação à turbidez (Figura 4.B), com valores de $\mathrm{pH}$ superior a 8,0 , obtiveram-se valores de turbidez inferiores à turbidez da água bruta, independente da concentração de coagulante aplicado. É notório que o diagrama de coagulação referente à cor apresenta melhores resultados em $\mathrm{pH}$ baixo, e o de turbidez, em $\mathrm{pH}$ mais elevado, coincidindo com os testes já feitos com este coagulante (BOMFIM, 2015; MORAES et al., 2007). 
Figura 3 - Diagramas de coagulação para o Ensaio 2 (Cloreto Férrico), Contendo isocurvas de cor aparente $(A)$ e turbidez (B)
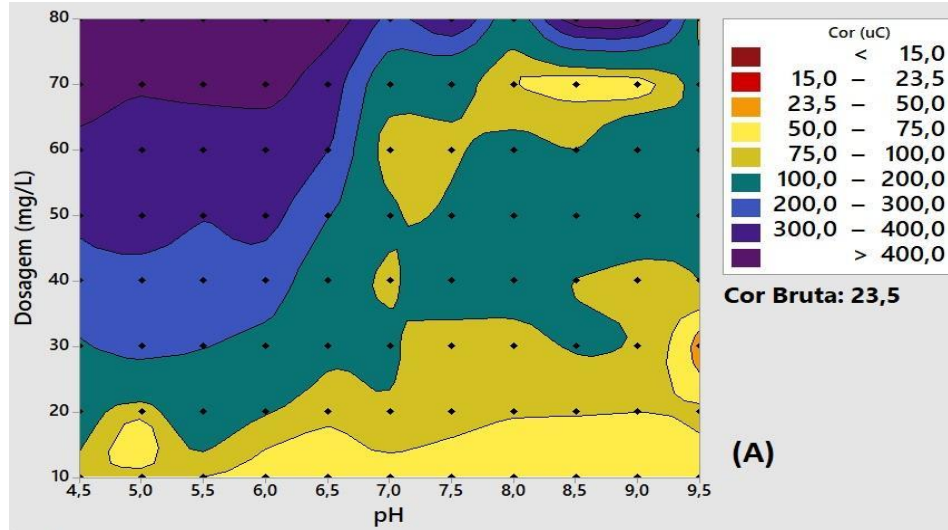

Cor Bruta: 23,5

(A)
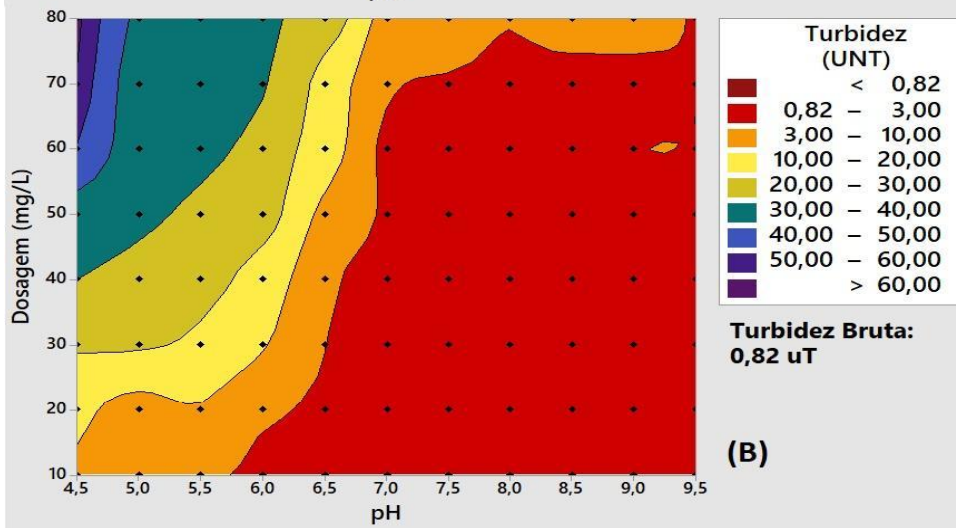

Fonte: Elaborada pelos autores

Figura 4 - Diagramas de coagulação para o Ensaio 3 (Tanfloc SG), contendo isocurvas de cor aparente $(A)$ e turbidez (B)
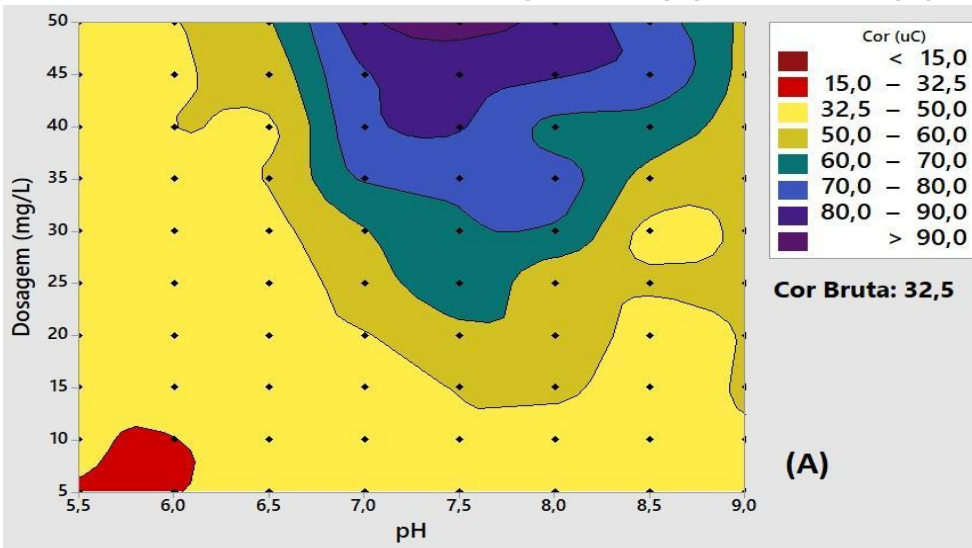

Cor Bruta: 32,5

(A)

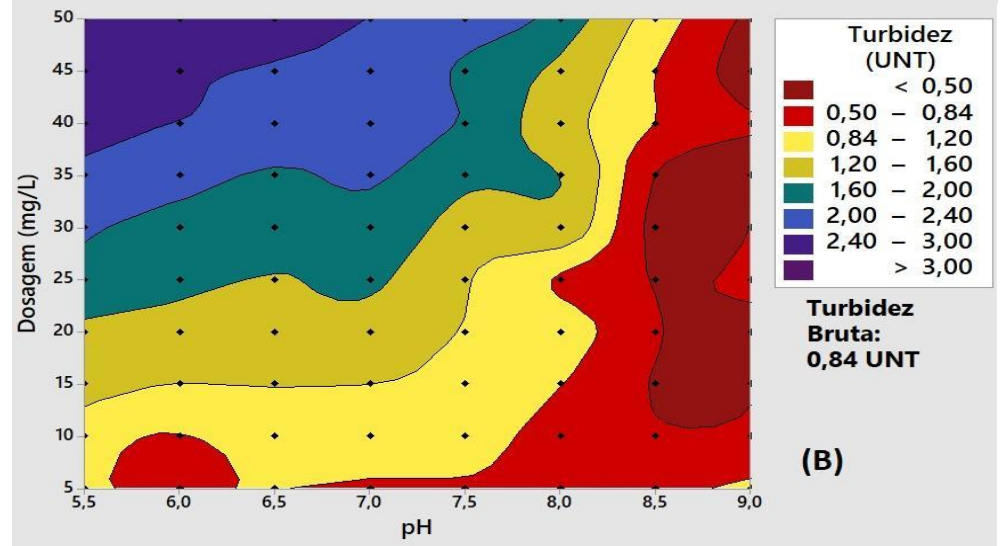

Fonte: Elaborada pelos autores. 
A elevação da cor pode ser explicada devido a uma maior formação de floco e maior solubilidade na água. Ressalta-se que os coagulantes taninos em dosagens mais elevadas podem conferir cor mais elevada, já que é a base de tanino que apresenta sua própria cor natural. Em ambos, porém, as baixas concentrações ( 5 e $10 \mathrm{mg} \mathrm{L}^{-1}$ ) obtiveram resultados satisfatórios.

Trevisan (2014) alcançou resultados de remoção de até $99 \%$ de cor aparente com o Tanfloc SG em água bruta para abastecimento, porém, utilizando tempo de floculação inferior ao adotado neste estudo. É possível que um alto tempo de floculação não seja ideal para a aplicação de Tanfloc SG, fazendo com que os flocos formados sejam quebrados, gerando cor na água. Outro fator que fortalece esta afirmação, verificada no mesmo estudo de Trevisan, é que à medida que o tempo de sedimentação adotado aumenta, menor é a remoção de cor aparente do experimento, sugerindo uma dissolução dos flocos na água com o passar do tempo.

Por fim, a Figura 5 ilustra os diagramas de coagulação para o ensaio com o agente orgânico Tanfloc SL.

\section{Figura 5 - Diagramas de coagulação para o Ensaio 3 (Tanfloc SL),} contendo isocurvas de cor aparente $(A)$ e turbidez (B)
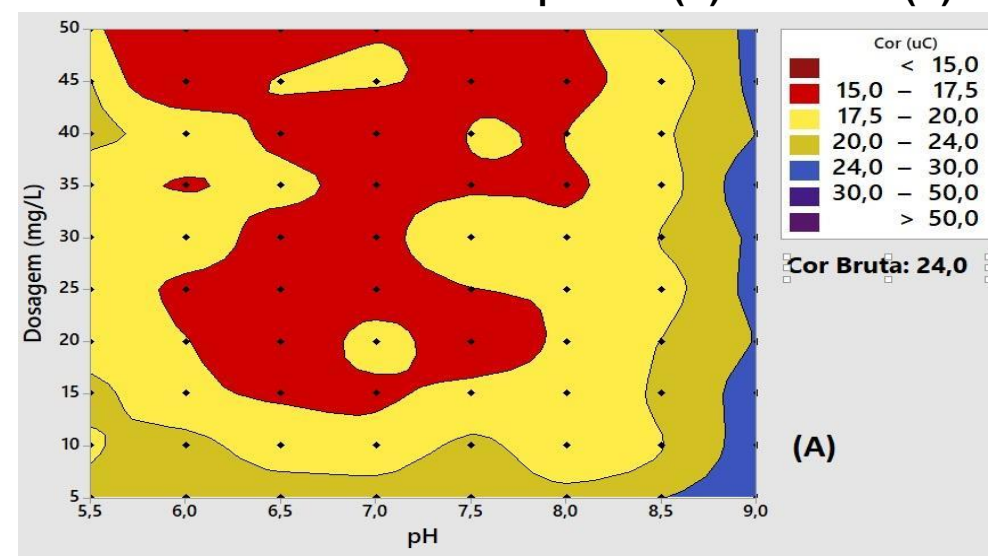

Cor Bruta: 24,0

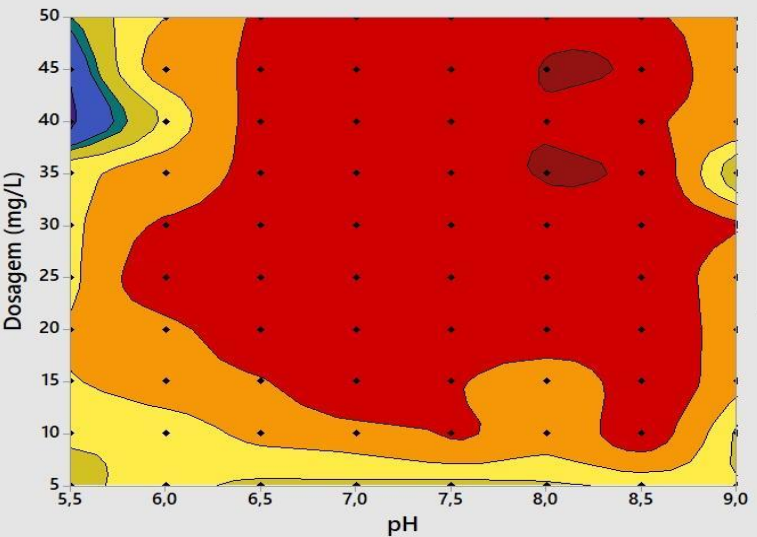

(A)

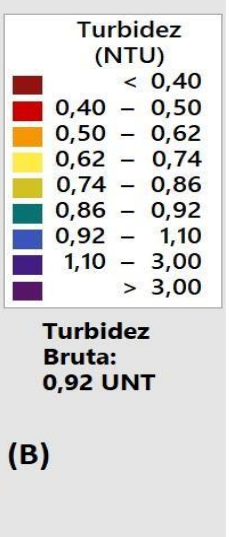

Fonte: Elaborada pelos autores.

Por meio dos diagramas do Tanfloc SL, é perceptível uma ampla faixa de $\mathrm{pH}, 6,0$ a 8,0, com resultados satisfatórios de remoção de turbidez e cor, porém, com o SL são necessárias dosagens mais elevadas de coagulante para atingir resultados semelhantes ao do Tanfloc SG. Apesar disso, todos os resultados de turbidez foram inferiores a 1,10 UNT, e grande parte destes foi inferior ao valor da turbidez da água bruta, com destaque aos valores de turbidez inferiores a 0,4 UT, que é um valor de turbidez inferior ao preconizado pela Portaria de Consolidação no 05/2017 do Ministério da Saúde, em seu anexo XX (BRASIL, 2017), após tratamento completo.

Os coagulantes orgânicos Tanfloc se comportam como polímeros orgânicos, prevalecendo o mecanismo de coagulação por pontes químicas, o que forma flocos densos que sedimentam rapidamente, sendo a velocidade de sedimentação adotada suficiente para remoção de boa parte dos sólidos em suspensão. Os coagulantes inorgânicos apresentaram deficiência em remover a turbidez e a cor presentes na água bruta, o que pode ser atribuído ao valor adotado para a velocidade de sedimentação, 
possivelmente insuficiente para sedimentação do floco formado durante a etapa de floculação. A partir da leitura dos diagramas, pode-se perceber ainda que, para os coagulantes inorgânicos, são necessárias baixas concentrações para um melhor resultado de turbidez e cor.

Moraes et al. (2007) verificaram a eficiência de remoção de cor e turbidez do Tanfloc SL utilizando-se água bruta do rio Pirapó, e observaram melhor eficácia em faixas de $\mathrm{pH}$ inferiores a 5,0. No presente estudo, os melhores resultados de remoção de cor e de turbidez foram obtidos em faixas de $\mathrm{pH}$ acima de 6,0.

\section{CONCLUSÃO}

Os diagramas de coagulação puderam traçar um perfil das melhores opções para o tratamento da água referente ao reservatório Epitácio Pessoa, podendo ser útil também para águas de características semelhantes, ou seja, baixa turbidez e cor aparente.

Foi possível verificar que os coagulantes inorgânicos tiveram pouca eficiência para remoção de turbidez e cor da água bruta, diferentemente dos orgânicos que possuíram uma maior eficiência para a remoção das impurezas. Atingiram-se valores de turbidez de até 0,4 UNT após a decantação, o que deixou a água em condições adequadas para a produção de águas filtradas com baixos índices de turbidez, podendo garantir maior remoção de microrganismos.

São necessários estudos posteriores de avaliação do custo-benefício para uma possível alteração e implantação de um novo coagulante no tratamento da água daquele reservatório.

\section{REFERÊNCIA}

AMIRTHARAJAH, A.; MILLS, M. Rapid-mix design for mechanisms of alum coagulation. Journal of the American Water Works Association, v. 74, n. 4, p. 210-216, 1982.

BARTIKO, D.; JULIO, M. DE. Construção e emprego de diagramas de coagulação como ferramenta para o monitoramento contínuo da floculação em águas de abastecimento. Revista Ambiente \& Água, v. 10, n. 1, p. 71-81, 2015.

BATAGHIN, F. A.; COSTA, M. A. B.; TAKAGAKI, J. Possibilidade de aplicação de tecnologias de tratamento de água das ETA's em usinas do setor sucroenergético no Estado de São Paulo. Revista Livre de Sustentabilidade e Empreendedorismo, v.
2, n. 3, p. 130-141, 2017.

BERNARDO, L. DI; DANTAS, A. D. B.; VOLTAN, P. E. $\mathrm{N}$. Tecnologias de tratamento, processos e operações. In: Tratabilidade de água e dos resíduos gerados em estações de tratamento de água. São Carlos: Editora LDIBE LTDA, 2011. p. 97-153.

SABOGAL PAZ, L. P. Seleção de tecnologias de tratamento de água. São Carlos, SP: Editora LDIBE LTDA, 2008.

BOMFIM, A. P. D. S. Avaliação dos coagulantes tanfloc em comparação aos coagulantes inorgânicos à base de alumínio no tratamento de água. 2015. 211 f. Dissertação (Mestrado) - Programa de Pós-Graduação em Tecnologia Ambiental, Universidade de Ribeirão Preto, Ribeirão Preto, 2015.

BRASIL. Ministério da Saúde. Portaria de Consolidação $n^{\circ} 5$ de 28 de setembro de 2017. Anexo XX. Consolidação das normas sobre as ações e os serviços de saúde do Sistema Único de Saúde, 2017.

Conselho Nacional do Meio Ambiente. Resolução CONAMA no 357 de 17 de março de 2005. Dispõe sobre a classificação dos corpos de água e diretrizes ambientais para o seu enquadramento, bem como estabelece as condições e padrões de lançamento de efluentes, e dá outras providências, 2005.

CRUZ, J. G.; MENEZES, J. C. S. S.; RUBIO, J.; SCHNEIDER, I. A. H. Aplicação de coagulante vegetal à base de tanino no tratamento por coagulação/floculação adsorção/coagulação/floculação do efluente de uma lavanderia industrial. In: CONGRESSO BRASILEIRO DE ENGENHARIA SANITÁRIA E AMBIENTAL, 23., 2005, Campo Grande, 2005. Anais... Campo Grande: CBESA, 2005

EDZWALD, J. K. (Ed.). Water quality \& treatment - a handbook on drinking water. 6. ed. USA: American Water Works Association and Mcgraw-Hill, 2011.

FARIAS, F. A.; FERREIRA, R. L.; FERRO, C. DE. Avaliação da eficiência de diferentes agentes coagulantes na remoção de cor e turbidez em efluente de fábrica de celulose não branqueada. Caderno Meio Ambiente e Sustentabilidade, v. 5, n. 3, p. 86-104, 2014.

FERRARI, T. N.; JULIO, M. DE; JULIO, T. S. DE; JÚNIOR, W. C. DE S. Estudos de tratabilidade das águas do Rio Paraíba do Sul que abastecem o município de São José dos Campos/SP. Revista AIDIS de Ingeniería y Ciencias Ambientales, v. 5, n. 2, p. 45-58, 2012. 
MANGRICH, A. S.; DOUMER, M. E.; MALLMANN, A. S.; WOLF, C. R. Química verde no tratamento de águas: Uso de coagulante derivado de tanino de acacia mearnsii. Revista Virtual de Quimica, v. 6, n. 1, p. 215, 2014.

MCLACHLAN, D. R. C. Aluminium and the risk for alzheimer's disease. Environmetrics, v. 6, p. 233-275, 1995.

MENESES, R. A. Diagnóstico operacional de sistemas de abastecimento de água: o caso de Campina Grande. 2011. 144 f. Dissertação (Mestrado em Engenharia Civil e Ambiental) - Universidade Federal de Campina Grande, Campina Grande, 2011.

MORAES, L. C. K.; BERGAMASCO, R.; TAVARES, C. R. G.; GIOVANI, M. C.; HENNIG, D. Avaliação da eficiência de remoção de cor e turbidez, utilizando como agente coagulante os taninos vegetais, com a finalidade de obtenção de água tratada. In: CONGRESSO BRASILEIRO DE ENGENHARIA SANITÁRIA E AMBIENTAL,, 24., 2007, Belo Horizonte. Anais... Belo Horizonte/MG: ABES, 2007

NEPOMUCENO, T. C. Estudo da aplicabilidade de coagulantes orgânicos e inorgânicos no tratamento de água para abastecimento público. 2016. $169 \mathrm{f}$. Trabalho de Conclusão de Curso (Graduação em Engenharia Sanitária e Ambiental) - Universidade Estadual da Paraíba, Campina Grande, 2016.

OLIVEIRA, T. F.; MIERZWA, J. C. Comparative performance evaluation of two UF pilot plants at the Alto da Boa Vista WTP (São Paulo, Brazil). Membrane Water Treatment, v. 2, n. 3, p. 175-185, 2011.

PARAÍBA. Plano Estadual de Recursos Hídricos. Resumo estendido, cap. 2. Caracterização fisiográfica e hidroclimática do estado da Paraíba, 2006.

PAVANELLI, G. Eficiência de diferentes tipos de coagulantes na coagulação, floculação e sedimentação de água com cor ou turbidez elevada. 2001. 233 f. Dissertação (Mestrado em Hidráulica e Saneamento) - Escola de Engenharia de São Carlos, Universidade de São Paulo, São Carlos,
2001.

PIANTÁ, C. A. V. Emprego de coagulantes orgânicos naturais como alternativa ao uso do sulfato de alumínio no tratamento de água. 2008. 74 f. Trabalho de Conclusão de Curso (Graduação em Engenharia Civil) - Universidade Federal do Rio Grande do Sul, Porto Alegre, 2008.

SANTOS, J. A. DOS. Caracterização socioeconômica e hídrica dos municípios da bacia do alto curso do Rio Paraíba - PB. [s.I.] Campina Grande: Universidade Estadual da Paraíba, 2014.

SHEN, Y. H. Treatment of low turbidity water by sweep coagulation using bentonite. Journal of Chemical Technology and Biotechnology, v. 80 , n. 5 , p. 581 586,2005

SILVA, C. DE M.; PORTO, C. DE A.; RODRIGUES, E. M. R.; SILVA, E. L.; JÚNIOR, R. H. DOS A. Prétratamento de efluente de laticínio visando à remoção da turbidez. In: CONGRESSO BRASILEIRO DE GESTÃO AMBIENTAL, 7., 2016, Campina Grande. Anais... Campina Grande-PB: IBEAS, 2016

SILVEIRA, T. N. Uso de diagramas de coagulação como ferramenta de avaliação de desempenho de coagulantes orgânicos e inorgânicos em águas naturais de características distintas. 2018. $41 \mathrm{f}$. Trabalho de Conclusão de Curso (Graduação em Engenharia Sanitária e Ambiental) - Universidade Estadual da Paraíba, Campina Grande, 2018.

TREVISAN, T. S. Coagulante Tanfloc SG como alternativa ao uso de coagulantes químicos no tratamento de água na ETA Cafezal. 2014. $106 \mathrm{f}$. Trabalho de Conclusão de Curso (Graduação em Engenharia Civil) - Universidade Tecnológica Federal do Paraná, Londrina, 2014.

WestPhalen, A. P. C.; CORÇÃO, G.; BenetTI, A. D. Utilização de carvão ativado biológico para o tratamento de água para consumo humano. Engenharia Sanitaria e Ambiental, v. 21, n. 3, p. 425436, 2016. 